Guy Le Boterf, Développer et mettre en œuvre la compétence : Comment investir dans le professionnalisme et les compétences

Le Boterf, G. (2018) Paris : Eyrolles.

Josée-Anne Côté

\title{
OpenEdition
}

Journals

Édition électronique

URL : https://journals.openedition.org/ripes/1565

DOI : 10.4000/ripes.1565

ISSN : 2076-8427

Éditeur

Association internationale de pédagogie universitaire

Référence électronique

Josée-Anne Côté, « Guy Le Boterf, Développer et mettre en œuvre la compétence : Comment investir dans le professionnalisme et les compétences ", Revue internationale de pédagogie de l'enseignement supérieur [En ligne], 34(3) | 2018, mis en ligne le 20 novembre 2018, consulté le 28 juin 2022. URL http://journals.openedition.org/ripes/1565 ; DOI : https://doi.org/10.4000/ripes.1565

Ce document a été généré automatiquement le 29 septembre 2020

Article L.111-1 du Code de la propriété intellectuelle. 


\section{Guy Le Boterf, Développer et mettre en œuvre la compétence : Comment investir dans le professionnalisme et les compétences}

Le Boterf, G. (2018) Paris : Eyrolles.

Josée-Anne Côté

\section{RÉFÉRENCE}

Développer et mettre en œuvre la compétence. Comment investir dans le professionnalisme et les compétences. [Le Boterf, G. (2018) Paris : Eyrolles.]

1 Depuis plusieurs années, l'enseignement supérieur s'inscrit dans une pédagogie axée sur les compétences, proposant des programmes à visées professionnalisantes notamment pour répondre aux attentes de la société et des industries et former des professionnels compétents. Bien que ce discours soit de plus en plus ancré dans le milieu de l'enseignement supérieur, qu'en est-il réellement de sa mise en place et de ses effets sur le terrain? Comment promouvoir cet esprit de professionnalisation dans les entreprises et les organisations? Comment reconnaître qu'un professionnel est compétent et comment développer cette compétence? Ce sont là des questions auxquelles se propose de répondre Guy Le Boterf ${ }^{1}$ dans son livre intitulé Développer et mettre en œuvre la compétence : Comment investir dans le professionnalisme et les compétences.

2 Le livre est divisé en neuf chapitres, dont le premier tente de déterminer les facteurs explicatifs de l'intérêt croissant pour le professionnalisme et la professionnalisation. Il y est, entre autres, fait état des attentes et des exigences des entreprises ou des organisations, ainsi que des clients, des usagers ou des patients envers les professionnels. Le deuxième chapitre décrit les apports, les limites et les risques de dérive des approches par compétences. L'auteur y présente la distinction qu'il fait entre 
«être compétent » et «avoir des compétences », distinction qui fonde sa définition « duale » de la compétence ${ }^{2}$ :

- Du point de vue "être compétent», il s'agit d'un processus consistant à «savoir agir en situation professionnelle en mobilisant une combinatoire appropriée de ressources internes personnelles (connaissances, savoir-faire ou habileté, aptitudes, émotions, ...) et externes (ressources de l'environnement) et en faisant appel à l'usage de fonctions de guidage ».

- Du point de vue "avoir des compétences », il s'agit d'une ressource personnelle «ou d'une combinaison de ressources personnelles nécessaires pour savoir agir en situation professionnelle» (p. 57)

3 C'est en voyant la compétence du point de vue « être compétent » que Le Boterf pose le troisième chapitre visant à définir ce qu'est un professionnel compétent en qui il est possible d'avoir confiance. Le quatrième chapitre propose de définir les fonctions de guidage, mentionnées dans la définition de la compétence du point de vue «être compétent », qui guident les processus amenant le professionnel à agir avec pertinence, compétence et éthique en situation. Le cinquième chapitre distingue trois niveaux de professionnalisme (débutant, professionnel confirmé et expert) et les caractéristiques qui permettent de les reconnaître dans le développement du professionnel. Le sixième chapitre s'attarde à la capacité de réflexivité, à son intérêt pour les professionnels et à la description des processus réflexifs. Cette réflexivité place le professionnel au cœur de son développement professionnel et de la mise en œuvre du professionnalisme qui est une responsabilité partagée ou conjointe, comme l'auteur le précise au septième chapitre. Dans le processus de développement du professionnel vient un moment où il faut se demander à quel point celui-ci est compétent. Le huitième chapitre présente des pistes pour l'évaluation du professionnalisme, que ce soit par rapport à l'entrée à utiliser pour cette évaluation ou au choix du dispositif d'évaluation proprement dit. Afin de favoriser la mise en œuvre de la compétence chez le professionnel, l'auteur suggère l'utilisation de parcours personnalisés de professionnalisation et le neuvième chapitre propose des suggestions pour la conception de ces parcours personnalisés.

Alors que le livre s'adresse clairement aux gestionnaires de ressources humaines d'entreprise ou d'organisation, il apporte aussi un éclairage sur les compétences pour l'enseignement supérieur dont l'une des missions est de préparer les étudiants à agir en professionnels compétents. L'auteur donne des exemples concrets concernant les attentes de la société et de l'industrie par rapport aux professionnels qui diplôment des institutions d'enseignement supérieur. La définition duale de la compétence proposée par Le Boterf apporte une vision qui permet de mieux se positionner sur ce qu'est la compétence, ce qui en soir devrait constituer la cible des formations en enseignement supérieur. Le livre met en lumière la place du professionnel lui-même dans son développement et l'importance de la réflexivité. Ces deux caractéristiques rappellent l'importance, dans les formations en enseignement supérieur, de mettre l'étudiant au centre de son apprentissage et de l'évaluation de sa compétence, en partenariat avec les enseignants. 


\section{NOTES}

1. Guy Le Boterf est directeur de Le Boterf Conseil (www.guyleboterf-conseil.com) et professeur associé à l'Université de Sherbrooke (Canada).

2. Les éléments constitutifs de cette définition ont été exposés dans ses ouvrages antérieurs mais ils sont ici synthétisés dans cette forme duale qui leur donne une nouvelle articulation.

\section{AUTEURS}

\section{JOSÉE-ANNE CÔTÉ}

Université de Sherbrooke, Sherbrooke, Canada

josee-anne.cote@usherbrooke.ca 